\title{
Missing physics in stick-slip dynamics of a model for peeling of an adhesive tape
}

\author{
Rumi De ${ }^{1, *}$ and G. Ananthakrishna ${ }^{1,2, \dagger}$ \\ ${ }^{1}$ Materials Research Centre, Indian Institute of Science, Bangalore-560012, India \\ ${ }^{2}$ Centre for Condensed Matter Theory, Indian Institute of Science, Bangalore-560012, India
}

\begin{abstract}
It is now known that the equations of motion for the contact point during peeling of an adhesive tape mounted on a roll introduced earlier are singular and do not support dynamical jumps across the two stable branches of the peel force function. By including the kinetic energy of the tape in the Lagrangian, we derive equations of motion that support stick-slip jumps as a natural consequence of the inherent dynamics. In the low mass limit, these equations reproduce solutions obtained using a differential-algebraic algorithm introduced for the earlier equations. Our analysis also shows that the mass of the ribbon has a strong influence on the nature of the dynamics.
\end{abstract}

It is well established that there are three different modes of failure during the peeling of an adhesive tape from a substrate [1]. At low applied velocities, the peeling front keeps pace with the pull velocity and the failure mode is cohesive. At high pull velocities, the failure is adhesive. In between these two regimes, there is an intermittent mode of failure corresponding to stick-slip dynamics [1,2] accompanied by a characteristic audible noise. The stick-slip nature suggests that this regime is unstable. Indeed, the strain energy release rate exhibits two stable branches separated by an unstable branch. Detailed studies by Maugis and Barquins [1] and others [3-5] show that the nature of the wave forms of the pull force exhibits sinusoidal, sawtooth, and highly irregular wave patterns. Gandur et al. [6] have carried out a dynamical analysis of the force wave forms and of acoustic emission signals, and report chaotic dynamics at the upper end of the pull velocities [6]. Analysis of acoustic signals has also been carried out by Ciccotti et al. [5].

Stick-slip behavior is commonly observed in a number of driven systems such as the Portevin-Le Chatelier (PLC) effect [7], frictional sliding [8], and earthquake dynamics [9]. Stick-slip is characterized by the system spending most part of the time in the stuck state and a short time in the slip state. One common feature of such systems is that the force exhibits "negative flow rate characteristic" (NFRC). In fact, studies of dynamics of such systems, including that of the adhesive tape, use the macroscopic phenomenological NFRC feature as an input, although the unstable region is not accessible.

Early studies by Maugis and Barquins [1] looked at the peeling problem from a dynamical angle. Later, a detailed study was carried out by Hong and Yue [3] on a threevariable model originally introduced by Maugis [10] using an " $N$ "-shaped function that mimics the peel force function. They report that the system of equations displayed periodic and chaotic stick-slip solutions. However, it was later recognized that the stick-slip oscillations were not obtained as a

\footnotetext{
*Electronic address: rumi@mrc.iisc.ernet.in

${ }^{\dagger}$ Electronic address: garani@mrc.iisc.ernet.in
}

natural consequence of the equations of motion $[4,11,12]$ as the jumps in the rupture speed were introduced externally once the velocity exceeded the limit of stability. Later, Ciccotti et al. [4] interpreted stick-slip jumps as catastrophes with the belief that the jumps in the rupture velocity could not be obtained from these equations of motion [4]. Recently we derived these equations starting from a Lagrangian and showed [11] that these equations are singular and fall in the category of differential-algebraic equations (DAE) [13] requiring a special algorithm. Using a DAE algorithm, we showed that stick-slip jumps across the two branches arise in a pure dynamical way. The dynamics was also shown to be much richer than anticipated earlier.

However, even as the DAE algorithm offers a mathematical framework for obtaining solutions for these singular equations, it is difficult to provide any physical interpretation for the "mass matrix" that removes the singularity. Thus, a proper identification of the missing physics responsible for the absence of dynamical jumps in these equations still remains to be addressed. As we shall comment later, this is a necessary step for understanding the origin of acoustic emission (AE) during peeling, a problem that has remained unresolved. A reexamination of the earlier derivation showed that the kinetic energy of the stretched part of the tape was ignored. Here, we show that the inclusion of this additional kinetic energy removes the singularity thus converting them into set of ordinary differential equations (ODE). Further, stick-slip jumps emerge as a natural consequence of the inherent dynamics itself. Apart from reproducing the DAE solutions for low mass limits, our analysis shows that the mass of the tape has a considerable influence on the nature of the dynamics.

A schematic of the experimental setup is shown in Fig. 1(a). An adhesive roller tape of radius $R$ is mounted on an axis passing through $O$ normal to the paper and is driven at a constant speed $V$ by a couple-meter motor positioned at $O^{\prime}$. The pull force $F$ acting along the line joining the contact point $P$ and $O^{\prime}$ subtends an angle $\theta$ to the tangent at the point $P$. As the contact point $P$ moving with a local velocity $v$ can undergo rapid bursts of velocity during rupture, the peeled length of the ribbon, $L=P O^{\prime}$ is not fixed. We note here that the peel force often called the force of adhesion (denoted by 


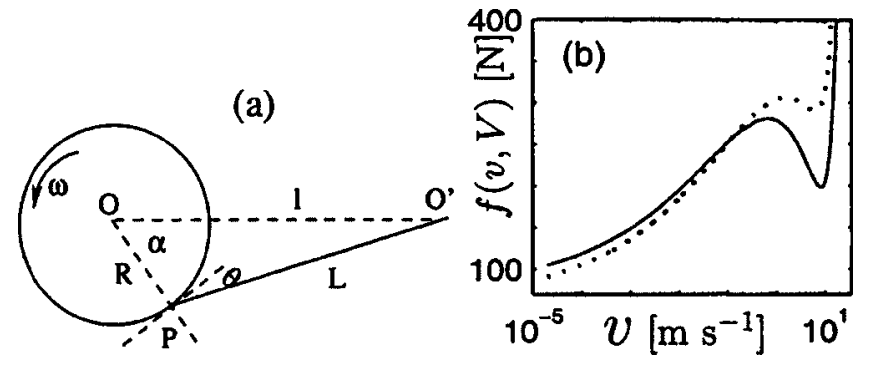

FIG. 1. (a) Schematic plot of an experimental setup. (b) The plots of $f(v, V)$ for $V=1$ (solid curve), $V=4$ (dotted curve).

$f$ ) is what is measured in experiments in steady-state conditions. Let the distance from the center of the roller tape $O$ to the motor $O^{\prime}$ be $l$ and $\alpha$ the angle subtended by $P O$ with the horizontal line $O O^{\prime}$. Let $I$ be the moment of inertia of the roller tape, $\omega$ the angular velocity, $k$ the elastic constant of the tape, and $u$ the elastic displacement of the tape. As the contact point is not fixed, $\omega=\dot{\alpha}+v / R$. The geometry of the setup gives $L \cos \theta=-l \sin \alpha$ and $L \sin \theta=l \cos \alpha-R$. As the peeling point $P$ moves, the pull velocity $V$ is the sum of three contributions [1], i.e., $V=v+\dot{u}-\dot{L}$, which gives

$$
v=V-\dot{u}+\dot{L}=V-\dot{u}-R(\cos \theta) \dot{\alpha} .
$$

Then, the Lagrangian is $\mathcal{L}=U_{K}-U_{P}$ with the kinetic energy given by $U_{K}=\frac{1}{2} I \omega^{2}+\frac{1}{2} m \dot{u}^{2}$ and the potential energy by $U_{P}$ $=\frac{1}{2} k u^{2}$. $\left[\frac{1}{2} I \omega^{2}\right.$ is the kinetic energy of the roller tape and $\frac{1}{2} m \dot{u}^{2}$, ignored earlier, arises due to the kinetic energy of the stretched part of the tape. $\dot{u}$ refers to the time derivative of $u$. In principle $m\left(m=m_{0} L(t)\right.$ where $m_{0}$ is the mass per unit length) depends on time through $L$. However, for all practical purposes $m$ can be treated as constant.] We write the dissipation function as $\mathcal{R}=\Phi(v, V)=\int f(v, V) d v$, where $f(v, V)$ is assumed to be derivable from a potential function $\Phi(v, V)$, physically represents the peel force, and is taken to depend on the pull velocity and rupture speed as in [11].

Using the Lagrange equations of motion,

$$
\begin{aligned}
& \frac{d}{d t}\left(\frac{\partial \mathcal{L}}{\partial \dot{\alpha}}\right)-\frac{\partial \mathcal{L}}{\partial \alpha}+\frac{\partial \mathcal{R}}{\partial \dot{\alpha}}=0, \\
& \frac{d}{d t}\left(\frac{\partial \mathcal{L}}{\partial \dot{u}}\right)-\frac{\partial \mathcal{L}}{\partial u}+\frac{\partial \mathcal{R}}{\partial \dot{u}}=0,
\end{aligned}
$$

and using $(\alpha, \dot{\alpha}, u, \dot{u})$ as the generalized coordinate, we get

$$
\begin{gathered}
\ddot{\alpha}=-\frac{\dot{v}}{R}+\frac{R}{I} \frac{\cos \theta}{(1-\cos \theta)} f(v, V), \\
m \ddot{u}=\frac{1}{(1-\cos \theta)}[f(v, V)-k u(1-\cos \theta)] .
\end{gathered}
$$

Note that the right-hand side of Eq. (3) is the algebraic constraint in Eq. (10) of Ref. [11] or Eq. (5d) of Ref. [3]. These equations in their present form are still not suitable for further analysis as they have to satisfy the constraint Eq. (1). In the spirit of classical mechanics of systems with constraints (see Ref. [14]), we derive the equation for the acceleration

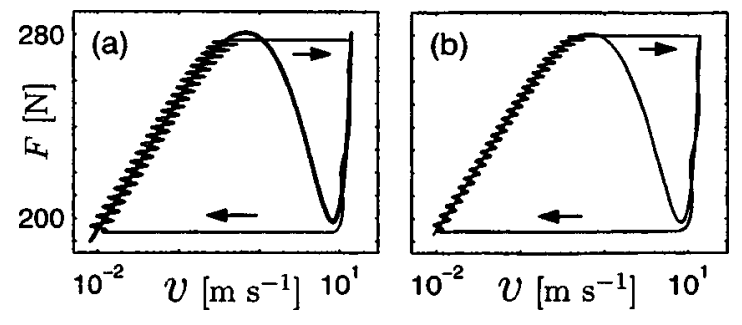

FIG. 2. (a), (b) The $v-F$ phase plots for $I=10^{-5}, V=1$ corresponding to the DAE and ODE solutions for $m=10^{-4}$. The solid line shows $f(v, 1)$.

variable $\dot{v}$ in the constraint equation by differentiating Eq. (1) and using Eqs. (2) and (3). This gives equations of motion for $\alpha, \omega, u$ and $v$

$$
\begin{gathered}
\dot{\alpha}=\omega-v / R, \\
\dot{\omega}=\frac{R}{I} \frac{\cos \theta}{(1-\cos \theta)} f(v, V), \\
\dot{u}=V-v-R(\cos \theta) \dot{\alpha}, \\
\text { Using Eqs. (2) and }(3) \text { in Eq. }(7), \text { we get } \\
\dot{v}=\frac{1}{(1-\cos \theta)}\left[\frac{k u}{m}-\frac{f(v, V)}{m(1-\cos \theta)}-\frac{(R \cos \theta)^{2} f(v, V)}{I(1-\cos \theta)}\right. \\
\left.+\frac{R}{L} \dot{\alpha}^{2}\left[l \cos \alpha-R(\cos \theta)^{2}\right]\right] .
\end{gathered}
$$

We retain the dynamization scheme introduced earlier [11] adopted from the PLC effect [15] wherein the difference between the maximum and minimum of $f(v, V)$ is assumed to decrease with increasing $V$. The parametrized form of $f(v, V)$ used here is given by

$$
\begin{aligned}
f(v, V)= & 402 v^{0.34}+171 v^{0.16}+68 e^{(v / 7.7)}-2 V^{1.5} \\
& -\left(415-45 V^{0.4}-0.35 V^{2.15}\right) v^{0.5} .
\end{aligned}
$$

Equation (9) mimics the general trend of the experimental peel force function and is essentially the same as used earlier [11] except that it accommodates larger excursions of trajectories arising from the introduction of the additional time scale [Fig. 1(b)]. The fixed point of Eqs. (4)-(6) and (8) given by $\alpha=0, \omega=V / R, u=f(V, V) / k$ and $v=V$ becomes unstable when $V$ is such that $f^{\prime}(V, V)<0$ leading to a Hopf bifurcation.

We have solved Eqs. (4)-(6) and (8) by adaptive step size stiff differential equations solver (MATLAB package) and studied the dynamics over a wide range of values of $I\left(\mathrm{~kg} \mathrm{~m}^{2}\right), V\left(\mathrm{~m} \mathrm{~s}^{-1}\right)$ for $m(\mathrm{~kg})$ ranging from $10^{-4}$ to 0.1 (keeping $k=1000 \mathrm{~N} \mathrm{~m}^{-1}, R=0.1 \mathrm{~m}$, and $l=1 \mathrm{~m}$ ). (Henceforth, we suppress the units for the sake of brevity.) Here, we present results (obtained after discarding the initial transients) for a few representative values of the $m$ when $(I, V)$ are at low and high values. We show that for low $m$, we 

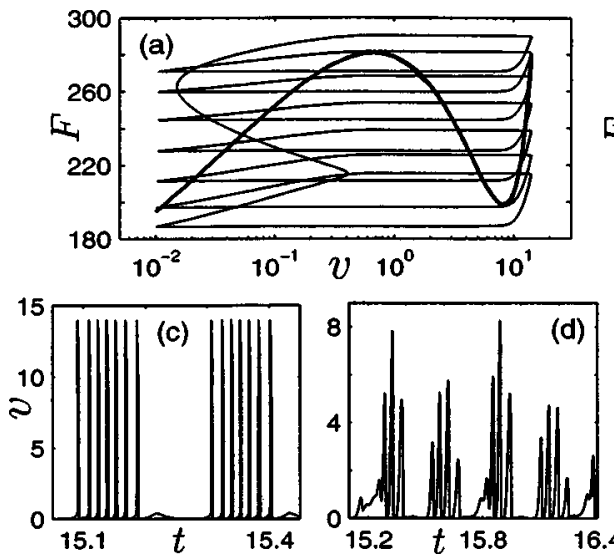
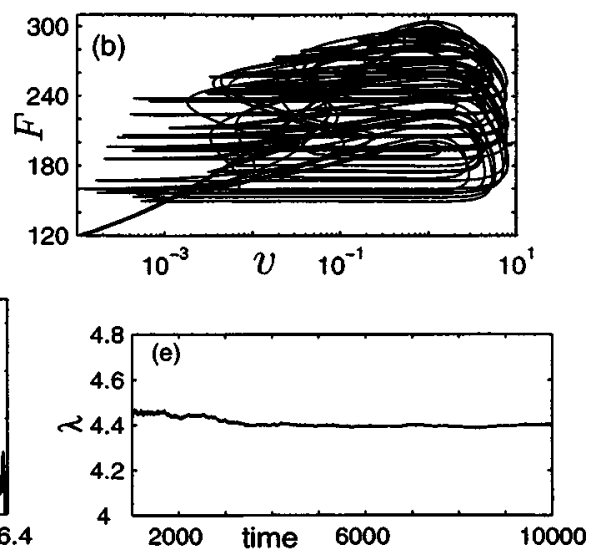

FIG. 3. (a), (b) Phase plots of $v-F$ obtained for $m=10^{-4}$ and 0.1 for $I=10^{-2}, V=1$. The solid line shows $f(v, 1)$. (c), (d) The plots of $v(t)$ for $m=10^{-4}$ and 0.1 . (e) The largest Lyapunov exponent, $\lambda$ for $m=0.1, I=10^{-2}, V=1$. (Units of $v$, $V$ are in $\mathrm{m} \mathrm{s}^{-1}, F$ in newtons, $I$ in $\mathrm{kg} \mathrm{m}^{2}, t$ in seconds, and $\lambda$ in $\mathrm{s}^{-1}$.) essentially recover the DAE solutions reported earlier [11]. Further we show that the mass of the tape has a strong influence on the nature of the dynamics.

A rough idea of the nature of the dynamics can be obtained by comparing the frequency $\Omega_{u}=(k / m)^{1 / 2}$ associated with $u$ with $\Omega_{\alpha}=(R f / I)^{1 / 2}$ corresponding to $\alpha$. Since $f$ (in newtons) is limited to $180-280$, the range of $\Omega_{\alpha}\left(\mathrm{s}^{-1}\right)$ is 1342-1673 for small $I\left(\sim 10^{-5}\right)$ decreasing to $42-53$ for large $I$ (0.01). In comparison, $\Omega_{u}\left(\mathrm{~s}^{-1}\right)$ is 3162 for $m=10^{-4} \mathrm{de}$ creasing to 100 for $m=0.1$. Thus, one expects the nature of solutions to be influenced with increasing $m$ for fixed $I$ which also depends of $V[11]$.

Here, we present our numerical results. Consider the low mass limit, i.e., low kinetic energy of the tape. Then the right-hand side of Eq. (3) is small, and turns $f(v, V)-k u(1$ $-\cos \theta) \approx f(v, V)-F(1-\cos \theta)=0$ (which is the algebraic constraint that makes the equations singular). Thus, one expects that the DAE solutions are reproduced for small $m$, which we have verified for the entire range of values of $I$ and $V$ studied previously [11]. (For numerical calculations, we have used $m=10^{-4}$ as the low mass limit.) As an example Figs. 2(a) and 2(b) show the phase plots in the $v-F$ plane obtained using the DAE algorithm and ODE Eqs. (4)-(6) and (8) for $m=10^{-4}$, respectively, keeping $I=10^{-5}, V=1$. It is evident that the DAE solution is similar to the ODE solution.

Much more complex dynamics emerges as a result of a competition between this additional time scale and other time scales present in the system. Consider the results for $m=10^{-4}$ and 0.1 , for $I=10^{-2}$, and $V=1$. The small $m$ plots are provided for the sake of comparison as they essentially correspond to the DAE solution. Consider the phase plots in $v-F$ plane shown in Fig. 3(a) for $m=10^{-4}$ and 3(b) for $m$ $=0.1$. It is clear that the influence of increasing $m$ is considerable. In particular, note that the sharp changes in $F$ in the $v-F$ plot [Fig. 3(a)] at the upper end of $v$ for small $m$ are rendered smooth for large $m$ case [Fig. 3(b)]. Indeed, the effect of the additional time scale due to the finite mass of the tape is also evident in the plots of $v(t)$ for the low and high mass cases shown in Figs. 3(c) and 3(d), respectively. Finally, it is clear that the phase plot [Fig. 3(b)] fills the space and is suggestive of chaotic dynamics. The chaotic nature can be ascertained by calculating the Lyapunov spectrum. Using the $Q R$ decomposition method [16], we have calculated the Lyapunov spectrum and find a large positive exponent with a value $\sim 4.4 \mathrm{~s}^{-1}[$ Fig. $3(\mathrm{e})]$.

Increasing $m$ does not always increase the level of complexity of the solutions. As an example, Figs. 4(a) and 4(b) show plots of $v(t)$ for $m=10^{-4}$ and 0.1 , respectively, for $I$ $=10^{-2}$ for $V=4$. While the solution for small $m$ is similar to that of DAE which exhibits several sharp spikes in velocity [Fig. 4(a)], for large mass $(m=0.1), v(t)$ is surprisingly simple and is periodic [Fig. 4(b)]. Indeed, this is better seen in the phase plots $v-F$ for $m=10^{-4}$ and 0.1 shown in Figs. $4(c)$ and 4(d) respectively. In contrast to the low mass $v-F$ plot, which is chaotic (see a similar DAE solution in Fig. 5(c) in Ref. [11]), a simple limit cycle emerges for $m=0.1$. As the nature of the dynamics can vary from a simple limit cycle to a chaotic attractor as the three parameters are varied, these results can be summarized as phase diagrams in the
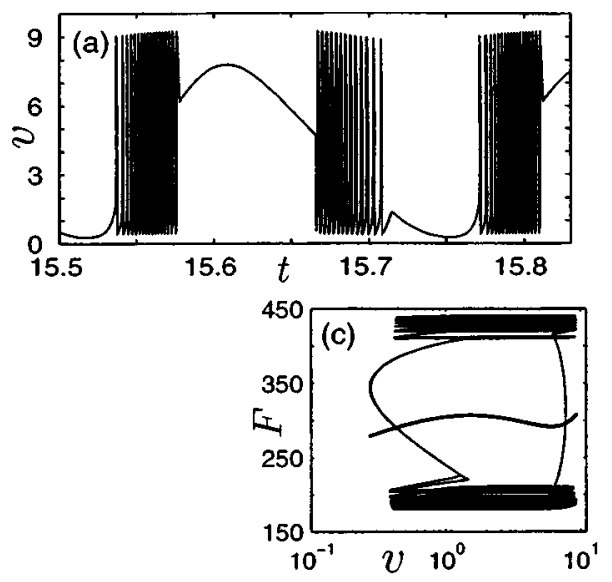
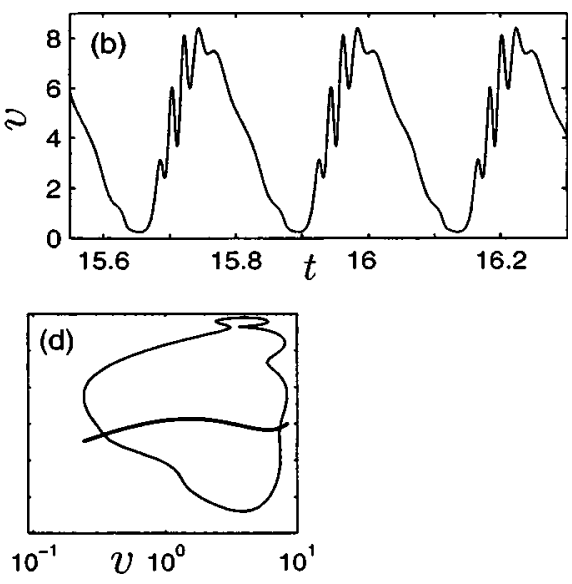

FIG. 4. (a), (b) Plot of $v(t)$ for $m=10^{-4}$ and 0.1 for $I=10^{-2}, V$ $=4$. (c), (d) The corresponding phase space trajectories. $f(v, V)$ is shown by a bold line. (Units of $v$, $V$ are in $\mathrm{m} \mathrm{s}^{-1}, F$ in newtons, $I$ in $\mathrm{kg} \mathrm{m}^{2}$ and $t$ in seconds.) 


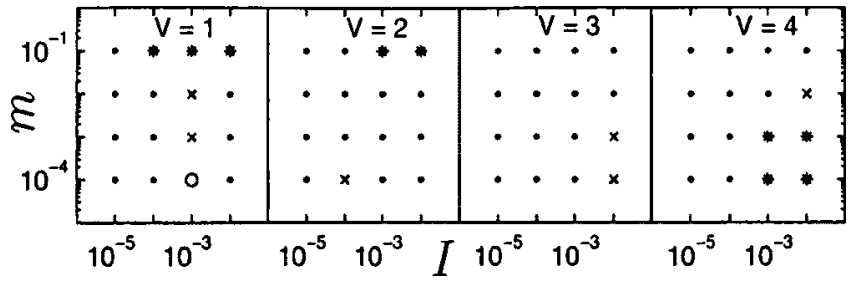

FIG. 5. Phase diagram in the $I-m$ plane for various values of $V$. Periodic $\bigcirc$, long periodic $\times$, chaotic (marginal) $\bigcirc$ and chaotic $*$. (Unit of $I$ is in $\mathrm{kg} \mathrm{m}^{2}$ and $m$ in $\mathrm{kg}$.)

$I-m$ plane for different values of pull velocities, $V$, as shown in Fig. 5. Apart from the chaotic state $(*)$ seen for a few values of the parameters, for most values, the system is periodic $(\bullet)$ and for a few other values, the attractor is long periodic $(X)$. We also find a (marginally) chaotic attractor (O) for $V=1, m=10^{-4}, I=10^{-3}$, for which the positive Lyapunov exponent is $\sim 0.03 \mathrm{~s}^{-1}$ (which is much beyond the error in computation).

In summary, we have demonstrated that the missing time scale arising from the kinetic energy of the stretched part of the tape plays an important role in the peeling dynamics of the adhesive tape. As the inclusion of this term lifts the singularity in the equations of motion hitherto considered, stickslip jumps across the two resistive branches emerge as a consequence of the inherent dynamics. Further, our study shows that the mass of the tape has a strong influence on the nature of the dynamics. For low pull velocities, and high $I$, the complexity increases, i.e., trajectories that are not chaotic for low mass become chaotic with increasing $m$. In contrast, for high $V$, the trajectories that are chaotic for low $m$ are rendered nonchaotic with increase of $m$.

Apart from resolving the central issue, the inclusion of kinetic energy of the tape provides a mechanism for converting the potential energy stored in the stretched tape into kinetic energy and hence provides a basis for explaining acoustic emission during peeling. This involves first extending the model to include the spatial degrees of freedom of the peel front and including an additional (rupture) velocity dependent dissipation function to mimic the AE energy dissipated (along the lines in [17]). The extended model also helps to analyze the contact line dynamics of the peeling front, a problem which is not well understood. Preliminary results [18] show that the energy dissipated occurs in bursts similar to the nature of $\mathrm{AE}$ signals seen in experiments [5]. Here, it is worthwhile to comment on the dynamization of the friction law. The physical origin of this can be attributed to the viscoelastic nature of the fluid, which in turn implies a frequency-dependent elastic constant. Thus as higher pull speed allows lesser time for internal relaxation to be complete, the viscoelastic fluid behaves much like an elastic solid. Clearly, a rigorous derivation of the peel force function from microscopic considerations that includes the effect of the viscoelastic glue at the contact point is needed to understand the dynamics appropriately.

R.D. wishes to thank Rangeet Bhattacharyya for many useful discussions. This work is financially supported by Department of Science and Technology-SP/S2k-26/98, India.
[1] D. Maugis and M. Barquins in Adhesion 12, edited by K. W. Allen (Elsevier, London, 1988), p. 205.

[2] D. W. Aubrey and M. Sheriff, J. Polym. Sci., Part A-1 18, 2597 (1980).

[3] D. C. Hong and S. Yue, Phys. Rev. Lett. 74, 254 (1995).

[4] M. Ciccotti, B. Giorgini, and M. Barquins, Int. J. Adhes. Adhes. 18, 35 (1998).

[5] M. Ciccotti, B. Giorgini, D. Villet, and M. Barquins, Int. J. Adhes. Adhes. 24, 143 (2004).

[6] M. C. Gandur, M. U. Kleinke, and F. Galembeck, J. Adhes. Sci. Technol. 11, 11 (1997).

[7] L. P. Kubin, C. Fressengeas, and G. Ananthakrishna, in Dislocations in Solids, edited by F. R. N. Nabarro and M. S. Duesbery (Elsevier Science, New York, 2002) Vol. 11, p. 101.

[8] B. M. J. Persson, Sliding Friction: Physical Principles and Applications, 2nd ed. (Springer, Heidelberg, 2000).

[9] P. Burridge and L. Knopoff, Bull. Seismol. Soc. Am. 57, 341
(1967).

[10] D. Maugis, CRNS Report, 1991 (unpublished).

[11] Rumi De, Anil Maybhate, and G. Ananthakrishna, Phys. Rev. E 70, 046223 (2004).

[12] D. C. Hong (private communication).

[13] E. Hairer, C. Lubich, and M. Roche, Numerical Solutions of Differential-algebraic Systems by Runge-Kutta Methods (Springer-Verlag, Berlin, 1989).

[14] E. C. G. Sudarshan and N. Mukunda, Classical Dynamics: A Modern Perspective (John Wiley and Sons, New York, 1974).

[15] L. P. Kubin, K. Chihab, and Y. Estrin, Acta Metall. 36, 2707 (1988).

[16] J. P. Eckmann and D. Ruelle, Rev. Mod. Phys. 57, 617 (1985).

[17] R. Ahluwalia and G. Ananthakrishna, Phys. Rev. Lett. 86, 4076 (2003); Rumi De and G. Ananthakrishna, Europhys. Lett. 66, 715 (2004).

[18] Rumi De and G. Ananthakrishna (unpublished). 